\title{
El sector de la salud frente a los desastres y el cambio climático en Cuba
}

\author{
Guillermo Mesa Ridel, ${ }^{1}$ Joel González García, ${ }^{1}$ María Cristina Reyes Fernández, ${ }^{1}$ \\ Duniesky Cintra Cala, ${ }^{2}$ Yudix Ferreiro Rodríguez ${ }^{1}$ y José Ernesto Betancourt \\ Lavastida ${ }^{3}$
}

Forma de citar Mesa Ridel G, González García J, Reyes Fernández MC, Cintra Cala D, Ferreiro Rodríguez Y, Betancourt Lavastida JE. El sector de la salud frente a los desastres y el cambio climático en Cuba. Rev Panam Salud Publica. 2018;42:e24. https://doi.org/10.26633/RPSP.2018.24

RESUMEN

Por su impacto sobre la salud humana y su capacidad para generar daños, los desastres son uno de los problemas globales que más preocupan a la comunidad internacional. Sin embargo, y pese a los esfuerzos realizados por numerosos sistemas de salud, aún no se ofrece la atención necesaria a la reducción de vulnerabilidades y la falta de estrategias que eviten o minimicen los riesgos. Por estar ubicada Cuba en una zona de peligro permanente de desastres, su sector de la salud ha logrado acumular una notable experiencia en cuanto a la gestión correctiva de los riesgos y el aseguramiento de escenarios futuros menos riesgosos, con la activa participación de la comunidad y un proceso planificado y organizado para enfrentar el impacto del cambio climático. En este trabajo se resumen algunas de estas experiencias y se describen los peligros, las vulnerabilidades y los riesgos de desastres en Cuba, su Sistema de Defensa Civil, y la planificación y la organización en el sector de la salud para la reducción de desastres, así como los principales efectos y desafíos del cambio climático en el sistema de salud. Las lecciones aprendidas y las buenas prácticas ratifican el papel determinante de los recursos humanos para reducir las vulnerabilidades; el mayor desafío es evitar o minimizar los riesgos, avanzar en la investigación y la preparación de los profesionales ante el cambio climático, y optimizar la organización de los sistemas y los servicios de salud del país.

Palabras clave Desastres; cambio climático; políticas de salud; Cuba.

Las crisis que afectan hoy al planeta se superponen unas a otras, se complementan y se refuerzan mutuamente. A los conflictos éticos, económicos, políticos y sociales se suman otros no menos complejos, como los civiles, los alimentarios y los del medioambiente, cuyo deterioro afecta a la vida humana.

\footnotetext{
Escuela Nacional de Salud Pública, La Habana, Cuba. Enviar la correspondencia a Guillermo Mesa Ridel, gmesa@infomed.sld.cu

2 Representación de la Organización Panamericana de la Salud, La Habana, Cuba.

3 Ministerio de Salud Pública, La Habana, Cuba.
}

El cambio climático está afectando a la salud de las personas. Los estudios sobre las variaciones y los cambios en el clima evidencian el aumento de la temperatura media, la elevación acelerada del nivel del mar, las modificaciones extremas en los regímenes de lluvia y en los hábitos de algunas especies animales, así como el incremento de desastres de magnitud y complejidad inigualables $(1,2)$. Sin embargo, en muchos países los sistemas de salud aún se concentran en acciones reactivas y prestan escasa atención a la reducción de las vulnerabilidades y la incorporación de estrategias preventivas sostenibles que eviten o minimicen los riesgos.

La aprobación del Marco de Acción de Sendai para la Reducción del Riesgo de Desastres 2015-2030 brindó una oportunidad única para concluir la evaluación del Marco de Acción de Hyogo, así como para buscar soluciones consensuadas y eficientes a los desastres y los riesgos de múltiples orígenes en el contexto del desarrollo sostenible (3).

En este trabajo se resumen algunas de las experiencias del sector de la salud cubano en el enfrentamiento a los desastres y las consecuencias del cambio climático

Este es un artículo de acceso abierto distribuido bajo los términos de la licencia Creative Commons Attribution-NonCommercial-NoDerivs 3.0 IGO, que permite su uso, distribución y reproducción en cualquier medio, siempre que el trabajo original se cite de la manera adecuada. No se permiten modificaciones a los artículos ni su uso comercial. Al reproducir un artículo no debe haber ningún indicio de que la OPS o el artículo avalan a una organización o un producto específico. El uso del logo de la OPS no está permitido. Esta leyenda debe conservarse, junto con la URL original del artículo. 
y se describen los peligros, las vulnerabilidades y los riesgos de desastres en Cuba, su Sistema de Defensa Civil, y la planificación y la organización en el sector de la salud para la reducción de desastres; se describen también los principales efectos y desafíos del cambio climático en el sistema de salud.

\section{PELIGROS, VULNERABILIDADES Y RIESGOS DE DESASTRES}

El análisis de los peligros o amenazas de desastres es la base para la identificación de las vulnerabilidades y la evaluación del riesgo.

Debido a su ubicación geográfica en el mar Caribe, Cuba comparte con los países de esta área el peligro recurrente de sufrir eventos de origen hidrometeorológico - formación y travesía de ciclones tropicales, lluvias intensas, inundaciones, tormentas locales de gran magnitud y sequías-, causantes de grandes pérdidas materiales y económicas, así como de afectaciones a la salud humana (4). Entre los años 1926 y 1944, la cifra de víctimas mortales por causa de los ciclones tropicales en Cuba fue de 3935 personas; los huracanes más destructivos fueron el ciclón de octubre de 1926, que afectó a la entonces Isla de Pinos (actualmente Isla de la Juventud) y La Habana, con 583 fallecidos; el huracán de noviembre de 1932 que arrasó con Santa Cruz del Sur, en la provincia de Camagüey, con 3033 fallecidos; y el ciclón de octubre de 1944, que pasó por Isla de Pinos y La Habana, y que causó 319 muertes. En octubre de 1963, otro evento devastador, el huracán Flora, dejó más de 1200 muertos en la región oriental del país, miles de damnificados y cuantiosos daños a la agricultura, las viviendas y la infraestructura del país (5).

En los últimos 20 años, Cuba ha recibido el impacto de 30 grandes eventos atmosféricos: 10 tormentas tropicales $\mathrm{y}$ 20 huracanes; de estos últimos, 11 fueron de gran intensidad (cuadro 1) y dejaron un saldo de 54 fallecidos y daños por más de US\$30000 millones en la infraestructura habitacional, la agricultura y la industria. Gracias al aviso estructurado a partir del sistema de alerta temprana para ciclones tropicales (6) y las medidas adoptadas de protección a la población, el país ha logrado reducir el número de víctimas humanas por esta causa.

Cuba también está expuesta a peligros de origen geológico, especialmente en la región suroriental del país, debido a la

CUADRO 1. Número de fallecidos y personas protegidas en huracanes de gran intensidad. Cuba, 2001-2017

\begin{tabular}{lclcc}
\hline Huracán & Categoría $^{a}$ & \multicolumn{1}{c}{ Fecha } & Fallecidos & Personas protegidas \\
\hline Michelle & 4 & Noviembre, 2001 & 5 & 783259 \\
Charley & 3 & Agosto, 2004 & 4 & 232929 \\
Iván & 4 & Septiembre, 2004 & 0 & 2266066 \\
Dennis & 4 & Julio, 2005 & 17 & 1551667 \\
Wilma & 5 & Octubre, 2005 & 0 & 760168 \\
Gustav & 4 & Agosto, 2008 & 0 & 467579 \\
Ike & 4 & Septiembre, 2008 & 7 & 2615794 \\
Paloma & 4 & Noviembre, 2008 & 0 & 1319433 \\
Sandy & 3 & Octubre, 2012 & 11 & 343230 \\
Matthew & 4 & Octubre, 2016 & 0 & 1373595 \\
Irma & 4 & Septiembre, 2017 & 10 & 1863589 \\
\hline
\end{tabular}

a Según la escala Saffir-Simpson, que define y clasifica la categoría de un huracán de acuerdo con la velocidad de los vientos. Fuente: Elaborado con datos a partir de los informes resumen sobre eventos meteorológicos del Estado Mayor Nacional de la Defensa Civil.

actividad sísmica por el movimiento de las placas tectónicas (7). En el sismo de mayor intensidad —ocurrido en el año 1932 en la zona de Santiago de Cuba, con una magnitud de 6,75 en la escala de Richter-se registraron 20 muertos, más de 400 heridos y daños importantes en $80 \%$ de las edificaciones de la ciudad.

En el ámbito de los desastres antropogénicos sobresalen los tecnológicos, fundamentalmente por accidentes del transporte, tanto automotor como ferroviario $(8,9)$, y los accidentes químicos en industrias y zonas aledañas, ocasionados por derrames y escapes de sustancias tóxicas, como el ocurrido en 1990 por un derrame de amoníaco en la ciudad de Matanzas, capital de la provincia del mismo nombre (10).

Entre los peligros sanitarios, los más frecuentes son los provocados por epidemias, condicionadas por la situación epidemiológica internacional y las violaciones en la legislación sanitaria, sobre todo en los territorios que se caracterizan por condiciones higiénico-sanitarias desfavorables y baja percepción del riesgo en la población (11). Asimismo, existe la posibilidad de agresiones biológicas y otras amenazas (12).

Las principales vulnerabilidades existentes pueden clasificarse en: a) estructurales, que varían en dependencia del estado del fondo habitacional y del nivel de explotación o falta de mantenimiento preventivo; b) las no estructurales, dadas por la susceptibilidad de las líneas vitales (acueducto, alcantarillado, electricidad, comunicaciones, instalaciones para almacenamiento y vías de acceso); y c) la funcional, referida a la organización, la disponibilidad de recursos para satisfacer las necesidades de la atención médica, la transportación de pacientes, la hospitalización, el seguimiento y el control de las acciones, así como la percepción de riesgo, la preparación y el desempeño profesional (13-15).

El riesgo de desastres se refiere a las pérdidas esperadas por la incidencia de uno o varios peligros (simultáneos o concatenados), sobre uno o más elementos vulnerables en un momento dado, un lugar $\mathrm{y}$ unas condiciones determinadas. $\mathrm{Su}$ evaluación, cualitativa o cuantitativa, se realiza desde una perspectiva multidisciplinaria para cada uno de los desastres probables, según su recurrencia y posibles pérdidas tangibles e intangibles; estas evaluaciones de riesgo toman en cuenta no solo los posibles daños físicos a las estructuras, sino también las condiciones derivadas de la situación económica, financiera, alimentario-nutricional, higiénico-sanitaria, psicosocial, energética, de las comunicaciones y otras (16).

Todo ello requiere un enfoque holístico que facilite la comprensión de cada tipo de evento, y determine la naturaleza y las condiciones de vulnerabilidad de la población, los bienes, los servicios, los medios de sustento expuestos y el medioambiente. La toma de decisiones se basa en los principios siguientes: en primer lugar, proteger las vidas humanas y evitar las pérdidas económicas, y, en segundo lugar, garantizar la actividad de reducción de desastres al menor costo posible. De este análisis se derivan acciones preventivas para reducir las vulnerabilidades, mitigar los impactos y tomar las medidas de enfrentamiento para lograr un mínimo de afectaciones y una rápida recuperación. 


\section{EL SISTEMA DE DEFENSA CIVIL CUBANO}

Antes del año 1959 no existía un sistema eficiente de protección civil. La actividad se limitaba, básicamente, a las casas de socorro, dependientes del Ministerio de Salubridad, y a la actuación del cuerpo de bomberos, los cuales carecían de un respaldo gubernamental efectivo.

La actividad institucionalizada de defensa civil (DC) se creó en Cuba en 1962 bajo el nombre de Defensa Popular, con la misión principal de organizar la defensa de las ciudades y la protección de las industrias y los centros de servicios, con una base popular (17). Tras el impacto del huracán Flora en 1963, la Defensa Popular se fue perfeccionando hasta convertirse en un sistema de medidas de protección, de carácter estatal, dirigido a proteger a la población y sus bienes, la infraestructura y la economía nacional, tanto en condiciones consideradas normales como en situaciones de desastres y ante las consecuencias del cambio climático.

El presidente del Consejo de Estado dirige la DC a través del ministro de las Fuerzas Armadas Revolucionarias, que cuenta con un Estado Mayor Nacional para velar por el cumplimiento de las medidas y las normas relativas a la protección civil, los convenios internacionales, los programas de cooperación y la ayuda internacional en casos de desastres (figura 1) (18).

Cuando no hay ninguna emergencia, los presidentes de las asambleas provinciales y municipales del gobierno son los jefes a cada instancia para asegurar la marcha de la planificación y la organización de las medidas preventivas establecidas por la DC. A su vez, los jefes de los ministerios y otros organismos centrales del Estado, las entidades económicas y las organizaciones sociales son responsables, en sus respectivos niveles, de planificar y organizar las medidas de la DC, respaldados por normas jurídicas que disponen las responsabilidades de todos los componentes de la sociedad para la reducción de desastres y el enfrentamiento al cambio climático (19).

Ante la eventualidad de un desastre, se establecen los centros de dirección de los consejos de defensa, sobre la base de la estructura político-administrativa del país, con la participación de los órganos y los organismos estatales seleccionados; ellos dirigen y controlan el cumplimiento de las medidas de enfrentamiento que

\section{FIGURA 1. Estructura del Sistema de Defensa Civil de Cuba}

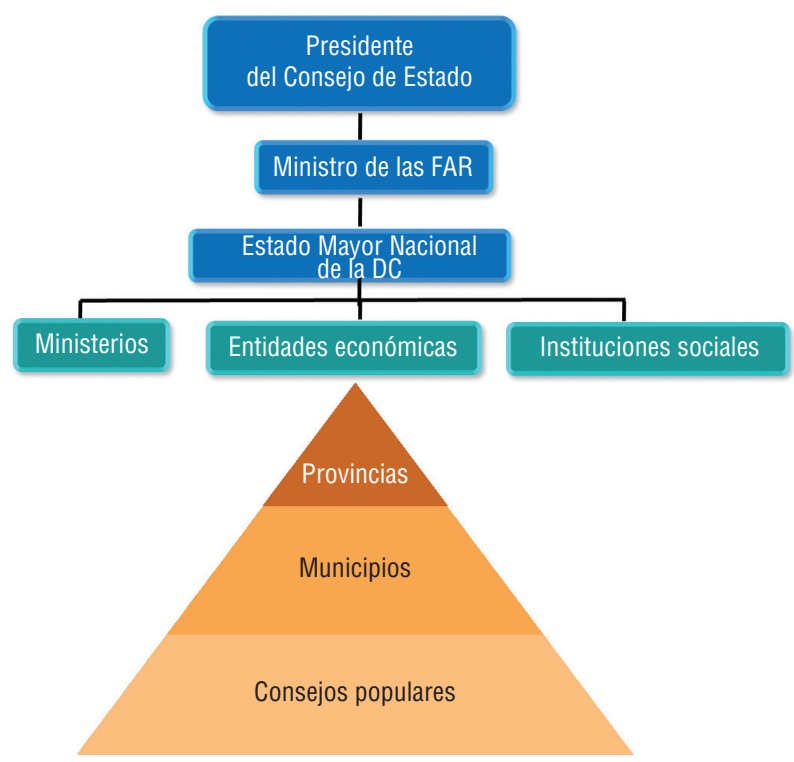

Nota: DC: Defensa Civil; FAR: Fuerzas Armadas Revolucionarias. Fuente: Elaborado por los autores a partir de la referencia 18.

\section{FIGURA2. Estructura de dirección para la respuesta y la recuperación ante desastres en Cuba}

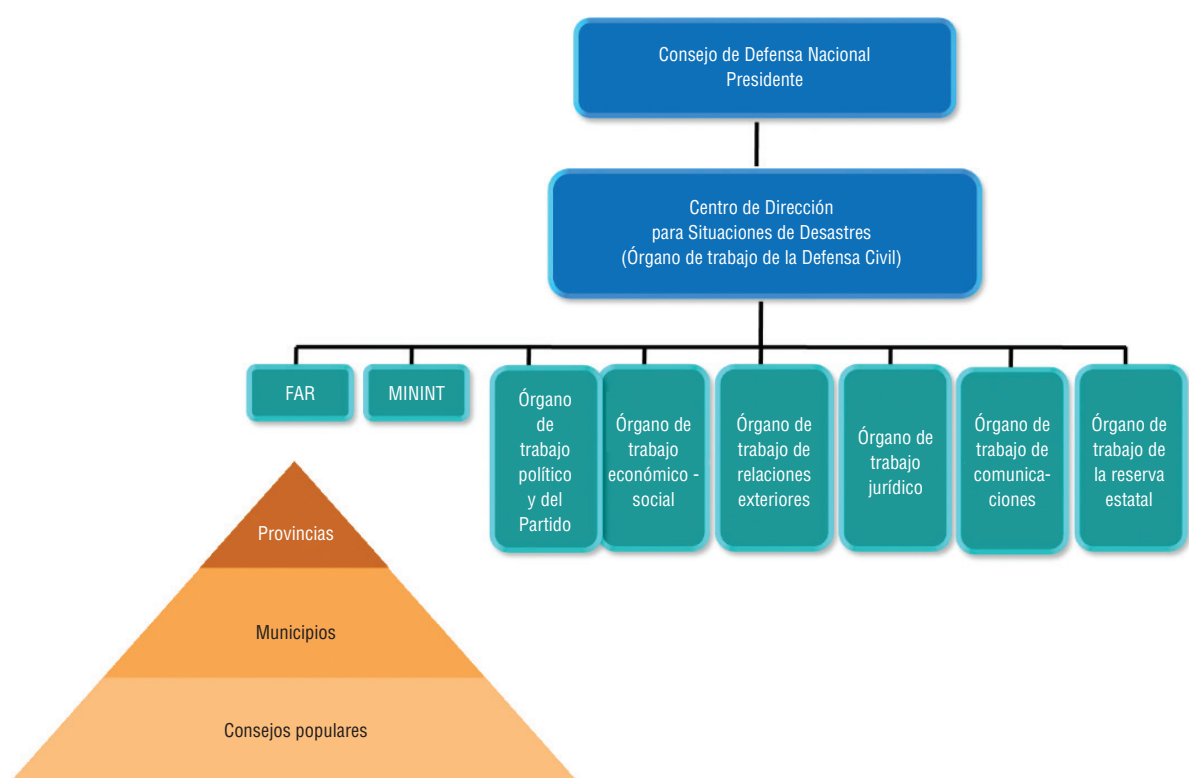

Nota: FAR: Fuerzas Armadas Revolucionarias; MININT: Ministerio del Interior. Fuente: Elaborado por los autores a partir de la referencia 18.

correspondan y mantienen un vínculo directo con las instancias de trabajo conformadas en los diferentes niveles para cumplir las decisiones relacionadas con la protección de las vidas y los recursos económicos de todo tipo (figura 2).

\section{El sector de la salud ante la reducción de desastres en Cuba}

El Ministerio de Salud Pública de Cuba forma parte del órgano de trabajo económico-social del Sistema Nacional de Salud a todos los niveles: municipal, provincial y nacional. Está facultado para emitir normativas relacionadas con la vigilancia, la prevención y el control de las enfermedades que pueden provocar epidemias. Asimismo, tiene el encargo de la preparación de la comunidad, a través de la Sociedad Nacional Cubana de la Cruz Roja, para el rescate ligero, los primeros auxilios, el socorrismo y el apoyo físico y psicológico a los damnificados. También 
elabora decisiones e indicaciones para las direcciones territoriales de la salud y sus instituciones, en las áreas de la asistencia médica y social, la higiene, la epidemiología y el aseguramiento logístico (20).

El plan de reducción de desastres es la herramienta básica para la planificación, la organización y la preparación del sector de la salud ante cualquier probabilidad de desastre (21). De acuerdo con ello, todas las direcciones territoriales e instituciones de la salud del país elaboran estos planes, fundamentados en las lecciones aprendidas en el enfrentamiento y la recuperación de los eventos ocurridos con anterioridad, los peligros identificados, el grado de vulnerabilidad y el nivel de riesgo. Las medidas, que disponen de respaldo económico, se organizan en cuatro etapas - prevención, preparativos, respuesta y recuperación (figura 3) — sobre la base de las fuerzas y los medios existentes; además, estas medidas deben ser medibles y actualizadas cada año. Las acciones que no se puedan ejecutar en el año en curso pasan a formar parte de las demandas de planificación a mediano plazo.

La etapa de prevención comienza tempranamente, desde el proceso inversionista y el planeamiento general del país, con el fin de evitar daños y pérdidas que puedan conducir a situaciones de

\section{FIGURA 3. Plan de reducción de desastres en Cuba: medidas por etapas}
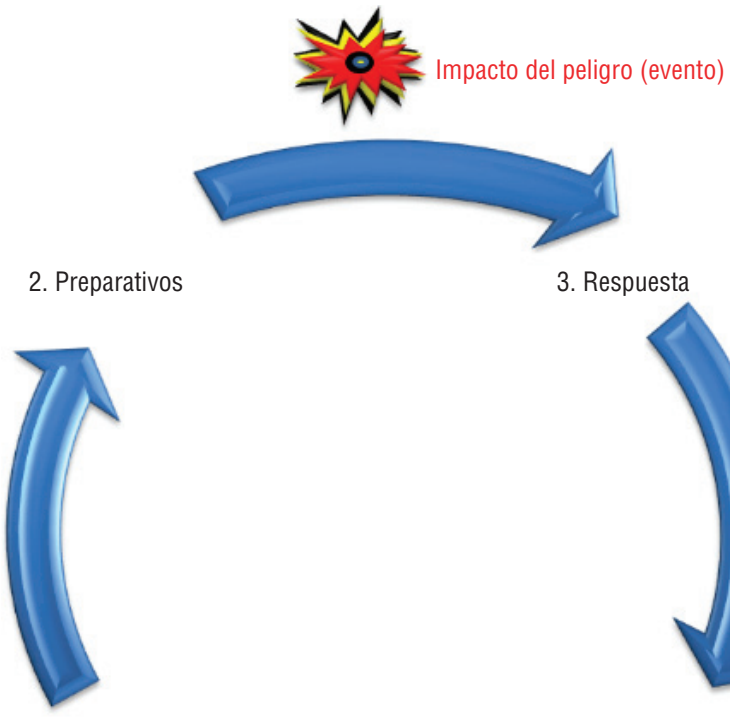

3. Respuesta

1. Prevención

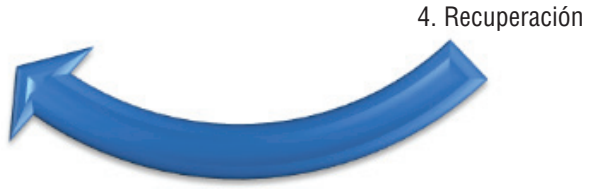

Fuente: Elaborado por los autores a partir de la referencia 18.
La etapa de preparativos está dirigida a organizar las capacidades, las fuerzas y los medios para una respuesta eficaz y un rápido restablecimiento de los servicios tras el impacto del evento. En esta etapa se toman en cuenta los resultados de los estudios de peligro, vulnerabilidad y riesgo territoriales (24) para organizar las medidas de protección y la evacuación de la población de áreas especialmente vulnerables y de alto riesgo hacia instituciones seguras; el despliegue anticipado de unidades médicas especializadas hacia los territorios que no dispongan de suficiente capacidad de respuesta o que puedan quedar aislados; así como la atención priorizada a las personas con enfermedades crónicas, discapacidad, de edad avanzada, embarazadas y niños. Comprende, además, la actualización de guías y manuales; la puntualización del abastecimiento; la protección de los medios, los equipos y los recursos; el traslado y la evacuación hacia albergues temporales; la limpieza de la red pluvial, las zanjas, los drenajes y los alcantarillados; y la poda de árboles, donde resulte necesario (25).

También se establece en esta etapa la preparación regular del personal de la salud y se completan y preparan las fuerzas para la respuesta: brigadas médicas ambulatorias, brigadas médicas quirúrgicas, grupos de salvamento y rescate de la Cruz Roja, grupos de manejo masivo de cadáveres, grupos de atención médica con especialistas en toxicología, directivos, profesionales, técnicos, trabajadores y estudiantes de la salud, así como las fuerzas especializadas de otros sectores, que comprende la atención psicológica (26).

La preparación de los profesionales del sector de la salud se inició en la década de 1970 con la incorporación de contenidos de Medicina de Desastre en el pregrado de las universidades médicas cubanas. Desde 1998, esta se convirtió en una asignatura curricular con esa denominación en todas las carreras universitarias de la salud y se extendió a la enseñanza de posgrado mediante cursos, talleres y diplomados en todas las provincias del país (27). También se instruye al personal docente y de salud para cumplir misiones en el exterior; se consolida la formación y la capacitación de estudiantes extranjeros con pasantías, cursos y diplomados; y se prepara a los estudiantes de la Escuela Latinoamericana de Medicina. Asimismo, se desarrolla la 
maestría Salud y Desastres, cuya cuarta edición se inició en el año 2017 (28).

La etapa de respuesta comienza ante la inminencia del impacto de un evento potencialmente destructivo, o cuando este ocurre. Durante esta etapa se cumplen las decisiones y los planes de reducción de desastres aprobados en cada instancia, en correspondencia con la fase decretada por la DC para cada territorio. En caso de una emergencia sanitaria, se establecen los planes de acción específicos anticipadamente, para contener las posibilidades de diseminación, en cooperación con los sectores de la economía que se requieran y con un alto componente de participación comunitaria.

La etapa de recuperación comienza a partir del momento en que ya no exista peligro o la situación que originó la respuesta esté controlada. Las primeras acciones están dirigidas a la rehabilitación de los servicios vitales y al restablecimiento de los elementos de apoyo a esos servicios y la infraestructura. Se establecen acciones de verificación, control, revisión, traslado y distribución de medios y recursos hacia las instituciones de salud, y se organiza la recepción, la distribución y el control de la ayuda externa. El proceso de recuperación y reconstrucción de las edificaciones e infraestructuras dañadas se realiza bajo el principio de no reproducir nuevas vulnerabilidades, es dirigida por el gobierno en sus diferentes instancias, y su duración depende del nivel de pérdidas y daños sufridos (19).

La aplicación del abordaje intersectorial ha sido una herramienta esencial de la práctica sanitaria para reducir los desastres, pues tiene una aplicación extensiva a los diferentes sectores de la sociedad (29). El sector de la salud recibe el apoyo de los órganos y los organismos del Estado y, a la vez, ofrece servicios mediante la coordinación y la concertación pactada entre las partes, lo que se manifiesta en el conocimiento de los peligros y de los sistemas de alerta temprana de las instituciones científicas; la evaluación de las vulnerabilidades y la identificación de las medidas de reducción de riesgo en instalaciones de la salud; la lucha contra vectores de enfermedades; el mantenimiento de la calidad sanitaria del agua de consumo humano y de los alimentos; la disponibilidad de medicamentos, medios médicos, transporte y comunicación; la atención médica al personal extranjero; la recepción de la ayuda internacional; y la divulgación de las medidas para elevar la educación sanitaria con la participación de la población (30).

Otro aspecto importante relacionado con la reducción de los efectos de los desastres en el que participa el sector de la salud ha sido la ayuda solidaria de Cuba a otros pueblos, que se inició en 1960 cuando se envió una brigada médica emergente y varias toneladas de equipos e insumos a Chile, afectado por un terremoto que causó miles de fallecidos. Desde entonces, más de 50 brigadas emergentes con 8023 colaboradores han asistido a 27 países dañados por terremotos, huracanes, inundaciones, epidemias o erupciones volcánicas. En el año 2005 se constituyó el Contingente Henry Reeve, oficialmente llamado Contingente Internacional de Médicos Especializados en Situaciones de Desastres y Graves Epidemias, cuyas brigadas han prestado ayuda a 19 países con 7217 colaboradores (cuadro 2), incluida la atención a 1000 enfermos de ébola en Guinea Conakri, Sierra Leona y Liberia durante la epidemia del 2014. En reconocimiento al trabajo y el altruismo de sus integrantes, la Organización Mundial de la Salud le otorgó a este contingente el Premio Memorial Lee Jong-wook (31).

\section{CUADRO 2. Participación del Contingente Internacional Henry Reeve en situaciones} de desastres, 2005-2016

\begin{tabular}{|c|c|c|c|}
\hline Año & País & Afectación & Número de colaboradores \\
\hline \multirow[t]{2}{*}{2005} & Guatemala & Inundaciones & 668 \\
\hline & Pakistán & Terremoto & 2564 \\
\hline \multirow[t]{2}{*}{2006} & Bolivia & Inundaciones & 602 \\
\hline & Indonesia & Terremoto & 135 \\
\hline \multirow[t]{3}{*}{2007} & Perú & Terremoto & 81 \\
\hline & Belice & Intensas Iluvias & 10 \\
\hline & México & Inundaciones & 54 \\
\hline 2008 & China & Terremoto & 43 \\
\hline 2009 & El Salvador & Inundaciones & 17 \\
\hline \multirow[t]{3}{*}{2010} & Haití & Terremoto & 1712 \\
\hline & Chile & Terremoto & 76 \\
\hline & Haití & Epidemia de cólera & 887 \\
\hline \multirow[t]{3}{*}{2014} & Sierra Leona & Epidemia de ébola & 165 \\
\hline & Guinea Conakri & Epidemia de ébola & 38 \\
\hline & Liberia & Epidemia de ébola & 54 \\
\hline \multirow[t]{4}{*}{2015} & Chile & Intensas Iluvias & 15 \\
\hline & Nepal & Terremoto & 48 \\
\hline & Dominica & Huracán & 15 \\
\hline & República Árabe Saharaui Democrática & Intensas Iluvias & 8 \\
\hline \multirow[t]{2}{*}{2016} & Fiji & Intensas Iluvias & 2 \\
\hline & Ecuador & Terremoto & 23 \\
\hline
\end{tabular}

Fuente: Elaborado con datos de la conferencia "Participación cubana en situaciones de desastres", del Dr. Iván Alexis Mora Pérez, vicedirector primero de la Unidad Central de Cooperación Médica, en el curso Preparativos para la Respuesta a Sismos de Gran Intensidad y Maremotos, dictada en La Habana, Cuba, del 11 al 13 de abril del 2017. 


\begin{tabular}{|c|c|}
\hline Logros & Retos \\
\hline $\begin{array}{l}\text { - Integración de la experiencia nacional e internacional sobre reducción de riesgos y } \\
\text { daños por desastres de todo tipo y su incorporación a la cooperación médica } \\
\text { internacional } \\
\text { - Elaboración de herramientas metodológicas de uso en la práctica sanitaria para la } \\
\text { reducción de los daños } \\
\text { - Asesoramiento mediante visitas técnicas a todas las provincias del país, para } \\
\text { mejorar el proceso de planificación, organización y preparación ante desastres } \\
\text { - Enfoque multidisciplinario, intersectorial y de participación social, con estricto } \\
\text { cumplimiento de la legislación cubana } \\
\text { - Superación profesional en salud y desastres: Esta ha sido una fortaleza } \\
\text { incuestionable para que el Sistema Nacional de Salud pueda minimizar las pérdidas } \\
\text { humanas y los daños por desastres a las instalaciones, aunque su implementación } \\
\text { en la práctica sanitaria es aún insuficiente } \\
\text { - Gestión de la información y la documentación, intercambio científico nacional e } \\
\text { internacional, y documentación de las estrategias y los resultados más valiosos; la } \\
\text { producción científica de los autores cubanos se compiló en } 10 \text { tomos de la serie } \\
\text { Salud y desastres: Experiencias cubanas (37) }\end{array}$ & $\begin{array}{l}\text { - Se necesita alcanzar una mayor comprensión de los riesgos de desastres } \\
\text { - Se debe sensibilizar más y formar mejor al personal de la salud como líderes } \\
\text { para mejorar y reducir la vulnerabilidad sanitaria } \\
\text { - Se debe involucrar más al personal de la salud en las estrategias de mitigación } \\
\text { y adaptación del sector al cambio climático } \\
\text { - Es necesario aprovechar más los beneficios del clima como procedimientos } \\
\text { terapéuticos } \\
\text { - Hay que aumentar el respaldo a la generación de evidencias científicas para } \\
\text { elevar la percepción del riesgo climático }\end{array}$ \\
\hline
\end{tabular}

en las que el pico epidémico estacional se movió de mayo a junio-julio, y de las epidemias más frecuentes de hepatitis viral tipo A, cuyo pico pasó de agostoseptiembre a los meses octubre-noviembre, con una nueva alza estacional en marzoabril. Además, se incrementó la densidad del vector Aedes aegypti (34), con mayor alcance espacial (en altura y extensión) y la aceleración de su ciclo reproductivo.

En abril del 2017, el Consejo de Ministros de Cuba aprobó el plan del Estado para el enfrentamiento al cambio climático, denominado Tarea Vida, que contempla cinco acciones estratégicas y 11 tareas orientadas a identificar y acometer acciones relacionadas con la protección costera de las ciudades; la relocalización de asentamientos humanos; la recuperación integral de playas, manglares y otros ecosistemas naturales protectores; el aseguramiento de la disponibilidad y el uso eficiente del agua; la disminución del deterioro, la rehabilitación y la conservación de los arrecifes de coral; y los proyectos vinculados con la energía renovable, la eficiencia energética, la seguridad alimentaria y los determinantes sociales y medioambientales de la salud (35).

El sector de la salud desarrolla un programa de enfrentamiento al cambio climático, planificado hasta el año 2030 (36). Este programa se enfoca en los ejes de investigación, capacitación, vigilancia y alerta temprana, a fin de actualizar la respuesta sectorial en correspondencia con el plan estatal. Su fin es elevar el conocimiento acerca de la influencia de la variabilidad climática en las enfermedades y la organización de los servicios de salud.

\section{ALGUNAS LECCIONES APRENDIDAS}

En el proceso de reducción de riesgos y daños por desastres de cualquier origen, el sector de la salud cubano ha alcanzado fortalezas que nacen de las buenas prácticas; pero también enfrenta dificultades. En el cuadro 3 se resumen las lecciones aprendidas.

\section{CONCLUSIONES}

A partir del trabajo realizado y las dificultades encontradas, se puede concluir que:

- La experiencia del sector de la salud cubano frente a los desastres hace hincapié en la gestión correctiva de los riesgos actuales y la construcción de escenarios futuros menos riesgosos, mediante una gestión planificada y organizada que incluye el enfrentamiento al cambio climático y la participación activa de toda la sociedad.

- Las lecciones aprendidas y las buenas prácticas ratifican el papel determinante de los recursos humanos para reducir las vulnerabilidades y respaldar las políticas y las estrategias dirigidas a evitar o minimizar los riesgos de desastres, un desafío que requiere atención permanente.

- La investigación en temas de salud y la preparación de los profesionales actuales y futuros para lograr una mitigación y adaptación más efectivas continúan siendo retos ante el impacto observado y esperado del cambio climático, así como para la organización de los sistemas y servicios de salud.

Agradecimientos. Los autores agradecen a Tania Borroto O’Farrill y Esther Paredes Esponda, por la ayuda brindada en la bibliografía; a Consuelo Macías, por las enseñanzas sobre redacción científica; a Wilfredo Cobas Dávila y Raúl Costa Gravalosa, por sus aportes a las cifras y los datos que se ofrecen; a José María Rubiera Torres, por sus sustanciales aportes al esclarecimiento de los hechos históricos; a Anaís García Fariñas, Leyla Sabina Blanco Pérez de Corcho y, en particular, a Gisele Coutin Marie, por sus oportunos comentarios, críticas y sugerencias a este trabajo.

\section{Conflicto de intereses. Ninguno.}

Declaración. Las opiniones expresadas en este artículo son responsabilidad de los autores y no reflejan necesariamente los criterios ni la política de la Revista Panamericana de Salud Pública / Pan American Journal of Public Health o de la Organización Panamericana de la Salud. 


\section{REFERENCIAS}

1. Organización Mundial de la Salud. Cambio climático y salud. Informe de la Secretaría. 62. ${ }^{\text {a }}$ Asamblea Mundial de la Salud. Ginebra: OMS; 2009. (Documento A62/11, punto 12.7). Disponible en: http://apps. who.int/gb/ebwha/pdf_files/A62/ A62_11-sp.pdf Acceso el 10 de septiembre de 2017.

2. Centella A, Bezanilla A. Escenarios climáticos. En: Planos E, Guevara AV, Rivero R, ed. Impactos del cambio climático y medidas de adaptación en Cuba. La Habana: Programa de las Naciones Unidas para el Desarrollo, Instituto de Meteorología; 2013. Pp. 99-115.

3. Oficina de las Naciones Unidas para la Reducción del Riesgo de Desastres. Marco de Sendai para la Reducción del Riesgo de Desastres 2015-2030. Ginebra: UNISDR; 2015. Disponible en: http://www.eird. org/americas/docs/43291_spanishsendaiframeworkfordisasterri.pdf Acceso el 10 de septiembre de 2017.

4. Pardo Gómez R, Rodríguez López Y. Clasificación de tormentas tropicales según lluvias asociadas: (1) estado del arte. RIHA. 2014;35(2):18-34. Disponible en: http:/ / scielo.sld.cu/pdf/riha/v35n2/ riha02214.pdf Acceso el 21 de noviembre de 2017.

5. Ramos Guadalupe LE. Huracanes: desastres naturales en Cuba. La Habana: Editorial Academia; 2009.

6. Rubiera Torres JM, Puig MA. The tropical cyclone early warning system in Cuba. En: Golnaraghi M. Institutional partnerships in multi-hazard early warning systems. A compilation of seven national good practices and guiding principles. Heidelberg: Springer Verlag; 2012. Pp. 9-28.

7. Chuy Rodríguez TJ. Acerca del impacto en el archipiélago cubano de los terremotos fuertes de las Antillas Mayores. En: Colectivo de autores. Salud y desastres. Experiencias cubanas (Tomo VIII). La Habana: Editorial Ciencias Médicas; 2014. Pp. 190-5.

8. Piña Tornés AA, González-Longoria Boada LB, Collejo Rosabal Y. Comportamiento epidemiológico de la mortalidad por accidentes de tránsito en Granma. 2005-2011. En: Colectivo de autores. Salud y desastres. Experiencias cubanas (Tomo X). La Habana: Editorial Ciencias Médicas; 2015. Pp. 156-63.

9. Villarroel Castro JM, Hernández Menéndez O, Barreras Rabaul D. La accidentalidad en los pasos a nivel de Cuba durante el período 1991-2007. Infraestructura Vial. 2008;(20):30-7. Disponible en: http://revistas.ucr.ac.cr/index.php/vial/article/ view/2031 Acceso el 21 de agosto de 2017.

10. Castro Hernández D, Luis Orozco JD, Curiel Lorenzo LD. Evaluación de atmósferas peligrosas en la zona industrial de Matanzas ante posibles accidentes químicos [CD-ROM]. Matanzas: Universidad de Matanzas Camilo Cienfuegos; 2013. Disponible en: http://monografias.umcc. cu/monos / 2013/Facultad \%20de \% 20 Ingenierias/mo13230.pdf Acceso el 12 de septiembre de 2017.
11. Guzmán MG, Peláez O, Kourí G, Quintana I, Vázquez S, Pentón M, et al. Caracterización final y lecciones de la epidemia de dengue 3 en Cuba 2001-2002. Rev Panam Salud Publica. 2006;19(4):282-9.

12. Turatis Manresa RM, Marrero Roque D, López Santa Cruz DI, Gómez Ochoa CA. Influencia del diferendo USA-Cuba: introducción de enfermedades infecciosas en Cuba. Rev Cubana Tecnol Salud. 2012;3(1). Disponible en: http://www.medigraphic. com/pdfs/revcubtecsal/cts-2012/ cts121d.pdf Acceso el 30 de agosto de 2017.

13. Llanes Burón C. Gestión de riesgo: una nueva visión de los desastres. En: Colectivo de autores. Salud y desastres. Experiencias cubanas (Tomo VI). La Habana: Editorial Ciencias Médicas; 2012. Pp. 31-7.

14. Vega Cuza I, Candebat Sánchez D, Oliva Álvarez R, Ferrera H. Estudios de vulnerabilidad sísmica estructural de las instalaciones hospitalarias: elemento imprescindible en la prevención de desastres. En: Colectivo de autores. Salud y desastres. Experiencias cubanas (Tomo VIII). La Habana: Editorial Ciencias Médicas; 2014. Pp. 27-36.

15. Candebat Sánchez D, Vega Cuza I. Vulnerabilidad sísmica no estructural. Importancia de su análisis para la mitigación de desastres en instalaciones de salud. En: Colectivo de autores. Salud y desastres. Experiencias cubanas (Tomo VIII). La Habana: Editorial Ciencias Médicas; 2014. Pp. 37-45.

16. República de Cuba, Estado Mayor Nacional de la Defensa Civil. Metodología para la organización del proceso de reducción de desastres, procedimientos para evaluar el nivel de reducción de la vulnerabilidad y el riesgo en los organismos, entidades y territorios; así como la objetividad de la implementación de los planes de reducción de desastres. La Habana: Estado Mayor Nacional de la Defensa Civil; 2017.

17. Castellanos A. Experiencia cubana en relación con los desastres. En: Colectivo de autores. Salud y desastres. Experiencias cubanas (Tomo VI). La Habana: Editorial Ciencias Médicas; 2012. Pp. 100-7.

18. República de Cuba, Consejo de Defensa Nacional. El Sistema de la Defensa Civil cubana. La Habana: Cuba Defensa. Disponible en: http://www.cubadefensa. $\mathrm{cu} / ? \mathrm{q}=$ =sistema-defensa-civil. Acceso el 12 de enero de 2018.

19. Castellanos A. Los desastres y fundamentos de la protección contra los mismos. En: Colectivo de autores. Salud y desastres. Experiencias cubanas (Tomo VI). La Habana: Editorial Ciencias Médicas; 2012. Pp. 73-99.

20. República de Cuba, Consejo de Defensa Nacional. Directiva N. ${ }^{\circ} 1$ del Presidente del Consejo de Defensa Nacional para la planificación, organización y preparación del país para las situaciones de desastres. La Habana: CDN; 2010. Disponible en: http:/ / www.sld.cu/galerias/pdf/sitios/desastres/directiva_vp_cdn_sobre_desastres.ultima_version.pdf Acceso el 30 de agosto de 2017.
21. Sauchay Romero L, Mesa Ridel G, Sánchez Gil Y, Rodríguez Fonseca P, Reyes Fernández MC. Plan de reducción de desastres: herramienta eficaz para la reducción de riesgos. En: Colectivo de autores. Salud y desastres. Experiencias cubanas (Tomo VIII). La Habana: Editorial Ciencias Médicas; 2014. Pp. 1-7.

22. República de Cuba, Comité Ejecutivo del Consejo de Ministros. Decreto No. 262/99. Reglamento para la compatibilización del desarrollo económico-social del país con los intereses de la defensa. La Habana: Consejo de Ministros; 1999. Disponible en: http://www.mvd.sld.cu/base_legal/ Decreto $\% 20 \% 20262 \% 20$ Compatibilizaion \%20de\%20desarrollo.pdf Acceso el 30 de agosto de 2017.

23. Gorry C. Approaches to climate change \& health in Cuba: interview to Guillermo Mesa MD MPhil, director, Disasters \& Health, National School of Public Health, Paulo Ortiz MS PhD, senior researcher, Climate Center, Cuban Meteorology Institute. MEDICC Review. 2015;17(2):6-9. Disponible en: http://medicc.org/mediccreview $/$ index.php issue $=34 \&$ id $=-$ 452\&a=vahtml Acceso el 21 de noviembre de 2017.

24. Programa de las Naciones Unidas para el Desarrollo, Agencia de Medio Ambiente. Metodologías para la determinación de riesgos a nivel territorial, P 1. La Habana: PNUD, AMA; 2014. Disponible en: http:/ / dipecholac.net/docs / files / 475-librometodologia-riesgo-ama.pdf Acceso el 21 de septiembre de 2017.

25. Navarro Machado VR. Situaciones de desastres. Manual para la organización de la atención médica de urgencias. 2. ${ }^{a}$ ed. La Habana: Editorial Ciencias Médicas; 2009. Disponible en: https:/ / www.researchgate. net/profile/Victor_Navarro_Machado / publication/293652479_Situaciones_de_ desastres_Manual_para_la_organizacion_ de_la_atencion_medica_de_urgencia/ links/56ba3a7808ae6a0040ade303/ Situaciones-de-desastres-Manual-para-laorganizacion-de-la-atencion-medica-deurgencia.pdf Acceso el 21 de noviembre de 2017.

26. Sánchez Gil YY, Lorenzo Ruiz A, Ventura Velázquez RE, Martínez Gómez C, Balseiro Estévez J. Estrategia de implementación de los lineamientos sobre la salud mental en situaciones de desastres en Cuba. En: Colectivo de autores. Salud y desastres. Experiencias cubanas (Tomo VIII). La Habana: Editorial Ciencias Médicas; 2014. Pp. 69-76.

27. Bello Gutiérrez B. La medicina de desastres, su inclusión en el programa de formación del médico general integral en Cuba. Panorama Cuba Salud. 2011;6 (2-3):26-31.

28. Ferreiro Rodríguez Y, Mesa Ridel G, Sánchez Gil Y, Sauchay Romero L, Gómez Miranda LL, Roca Ortiz EM. Un quinquenio de experiencias en la formación de postgrado en el CLAMED (2008-2012). En: Colectivo de autores. Salud y desastres. Experiencias 
cubanas (Tomo VIII). La Habana: Editorial Ciencias Médicas; 2014. Pp. 61-8.

29. Castell-Florit Serrate P. La intersectorialidad en la práctica social. La Habana: Editorial Ciencias Médicas; 2007.

30. Reyes Fernández MC, Mesa Ridel G. Instituciones de salud seguras. Experiencias alcanzadas. Cuba 2009. En: Colectivo de autores. Salud y desastres. Experiencias cubanas (Tomo IV). La Habana: Editorial Ciencias Médicas; 2011. Pp. 29-34.

31. Organización Mundial de la Salud, Centro de Prensa. Premio de Salud Pública en Memoria del Dr. Lee Jong-Wook 2017 al Contingente Henry Reeve (Cuba). 70. ${ }^{\text {a }}$ Asamblea Mundial de la Salud, Ginebra, 2017 mayo 26. Disponible en: http:/ / www. who.int/mediacentre/events / 2017 / wha70/lee-jong-wook-prize/es/ Acceso el 1 de septiembre de 2017.

32. Ortiz Bultó PL, Pérez EA, Rivero AV, Pérez $\mathrm{AC}$, Vázquez JRC, Guevara $\mathrm{AV}$, et al. Impactos de la variabilidad y el cambio climático en el sector de la salud, proyecciones al 2050 en Cuba. Rev Colomb Meteorol. 2010;(13):79-91.
33. Ortiz Bultó PL, Pérez A, Rivero A, Pérez A, Canga R. Salud humana. En: Planos E, Guevara AV, Rivero R, ed. Impactos del cambio climático y medidas de adaptación en Cuba. La Habana: Programa de las Naciones Unidas para el Desarrollo, Instituto de Meteorología; 2013. Disponible en: http:// research3.fit.edu/sealevelriselibrary/documents/doc_mgr/473/28.\%20Planos $\% 20$ et $\% 20$ al. $\% 20 \% 20(2012) . \% 20 \% 20$ Impacts $\% 20$ and $\% 20$ Adaptation $\% 20$ to $\% 20$ CC $\% 20$ in $\% 20$ Cuba.pdf Acceso el 21 de septiembre de 2017.

34. Ortiz Bultó PL, Rivero A, Linares Y, Pérez A. Spatial models for prediction and early warning of Aedes aegypti. Proliferation from data on climate change and variability in Cuba. MEDICC Review. 2015;17(2):20-8. Disponible en: http://medicc.org/mediccreview $/$ index.php issue $=34 \& \mathrm{id}=-$ $455 \& \mathrm{a}=\mathrm{vahtml}$ Acceso el 21 de noviembre de 2017.

35. República de Cuba, Consejo de Ministros. Tarea Vida. Plan de Estado para el enfrentamiento al cambio climático. La Habana: Ministerio de Ciencia, Tecnología y Medio Ambiente; 2017. Disponible en: https://
www.ecured.cu/Tarea_Vida Acceso el 21 de septiembre de 2017.

36. Guevara A, Paz L. República de Cuba. Segunda comunicación nacional a la Convención Marco de las Naciones Unidas sobre Cambio Climático. La Habana: Ministerio de Ciencia Tecnología y Medio Ambiente; 2015. Disponible en: http://unfccc.int/essential_background/ library/items /3599.php?rec $=$ j\&priref$=7803 \#$ \#beg Acceso el 2 de diciembre de 2017.

37. Colectivo de autores. Salud y desastres. Experiencias cubanas (Tomos I-X). La Habana: Editorial Ciencias Médicas; 2015. Disponible en: http://www.bvs.sld.cu/ libros/salud_desastre_x/indice_p.htm Acceso el 2 de diciembre de 2017.

Manuscrito recibido el 27 de abril de 2017. Aceptado para publicación, tras revisión, el 19 de octubre de 2017.
ABSTRACT

\section{The health sector in the face of disasters and climate change in Cuba}

Due to its impact on human health and its capacity to cause damage, disasters are one of the global problems that most concern the international community. However, and in spite of the efforts made by many health systems, the reduction of vulnerabilities and the lack of strategies to avoid or minimize risks have not received enough attention yet. As Cuba is located in an area of permanent danger of disasters, its health sector has accumulated considerable experience in the management of risks and in the assurance of less risky future scenarios, with active participation of the community and a planned and organized process to address the impact of climate change. This paper summarizes some of these experiences and describes the dangers, vulnerabilities, and risks of disasters in Cuba, its Civil Defense System, and planning and organization in the health sector for disaster reduction, as well as the main effects and challenges of climate change in the health system. Lessons learned and good practices support the key role of human resources to reduce vulnerabilities; the greatest challenges are to avoid or minimize risks, to advance research, to train professionals for climate change, and to optimize the organization of health systems and services in the country.

Keywords Disasters; climate change; health policy; Cuba. 
RESUMO Devido ao seu impacto na saúde humana e na sua capacidade de causar danos, os desastres são um dos problemas globais mais preocupantes para a comunidade internacional. No entanto, e apesar dos esforços feitos por numerosos sistemas de saúde, a

O setor da saúde diante de desastres e mudanças climáticas em Cuba atenção necessária ainda não foi oferecida para a redução de vulnerabilidades e a falta de estratégias que evitem ou minimizem os riscos. Como Cuba está localizada em uma área de permanente perigo de desastres, seu setor de saúde acumulou uma experiência considerável no gerenciamento de riscos e na garantia de cenários futuros menos arriscados, com a participação ativa da comunidade e um processo planejado e organizado para enfrentar o impacto das mudanças climáticas. Este artigo resume algumas dessas experiências e descreve os perigos, vulnerabilidades e riscos de desastres em Cuba, seu Sistema de Defesa Civil e planejamento e organização no setor da saúde para a redução de desastres, bem como os principais efeitos e desafios das mudanças climáticas no sistema de saúde. As lições aprendidas e as boas práticas ratificam o papel determinante dos recursos humanos para reduzir vulnerabilidades; o maior desafio é evitar ou minimizar riscos, avançar pesquisa, preparar profissionais para mudanças climáticas e otimizar a organização de sistemas e serviços de saúde no país.

Palavras-chave Desastres; mudança climática; política de saúde; Cuba. 